Gut, 1987, 28, S1, 45-49

\title{
Role of receptors in mediating trophic stimuli in the pancreas
}

\author{
JOHN A WILLIAMS \\ From the Cell Biology Laboratory, Department of Medicine, Mount Zion Hospital and Medical Center, \\ San Francisco, California, and the Departments of Physiology and Medicine, University of California, \\ San Francisco, California, USA
}

Pancreatic acinar cells synthesise and secrete a variety of digestive enzymes as well as a $\mathrm{NaCl}$-rich pancreatic juice. Acinar cells are able to adapt to the changing needs of the organism both by regulating the total pancreatic mass as well as by altering the synthesis of specific molecules. Adaptive regulation occurs with the onset of oral feeding at birth, the regrowth that follows surgical resection or destruction of the gland during pancreatitis, and as a change in content of specific digestive enzymes following a shift from one diet to another. ${ }^{1}$ Although not totally established, it is generally assumed that these adaptive responses are mediated by hormones or other regulatory molecules. Early work has focused on secretagogues such as cholecystokinin (CCK), acetylcholine and secretin. Cholecystokinin when injected into rats has been repeatedly shown to increase the mass and DNA and protein content of the gland. ${ }^{1}$ This makes teleological sense in that a secretory stimulus should enhance the synthesis of replacement digestive enzymes. Recently other regulatory molecules with more subtle or no secretory effects such as insulin, EGF, IGF, and corticosteroids have been shown to induce adaptive changes. While the different regulatory agents and mechanism of action involved in mediating growth are not well established these effectors are certain to interact with cellular receptors, therefore, it is clearly relevant to consider the properties of pancreatic receptors and how they could be involved in the initiation of a trophic response. Consideration of second messengers mediating intracellular responses will be mentioned when relevant but treated in less detail as they are covered elsewhere ${ }^{2}$ as well as in this volume.

\section{Functional characterisation of acinar receptors}

The function of regulatory molecules is limited to the

Address for correspondence: Dr John A Williams, Cell Biology Laboratory, Mount Zion Hospital and Medical Center, P.O. Box 7921, San Francisco, CA 94120, USA. transmission of information, therefore, a specific receptor protein exists and initiates the response to each regulatory molecule. Regulatory molecules of distinct chemical structure generally act on distinct receptors because the receptor is specific for a unique portion of the regulatory molecule. Regulatory molecules of similar structure - that is, CCK and gastrin, insulin and IGF may act on unique receptors, related receptors or the same receptor and this distinction is frequently difficult to make. In any case, regulatory molecules and their receptors can be characterised by their biological effects and more specifically by their mechanism of action. Regulatory molecules acting on pancreatic acinar cells fall into four general categories with regard to mechanisms of action (Table). Some of these regulatory molecules also affect pancreatic ducts where their biological response is different but the mechanism of action is probably similar.

In general the major regulators activating secretion of digestive enzymes are mediated intracellularly by calcium $\left(\mathrm{Ca}^{2+}\right)$ and diacylglycerol (DAG) while the major regulators of ductular fluid secretion are mediated by cyclic AMP (cAMP). ${ }^{2}$ This division is not absolute as cAMP mediated agents are well known to potentiate digestive enzyme secretion from acinar cells, while in some species such as the rat the $\mathrm{Ca}^{2+}$-DAG mediated agents also stimulate copious fluid secretion (although in this case the fluid may originate from acinar cells rather than ducts). Note that hormones and neurotransmitters are found in both groups and do not separate on the basis of mechanism of action. In addition some receptors originally described as responding to amphibian derived peptides are now known to be activated by endogenous mammalian peptides. In these cases the homologous mammalian peptide is listed first in the Table as for example 'GRP, bombesin'. While most of these regulators are excitatory, at least one inhibitory regulator exists, somatostatin, which in the pancreas, as in other target cells, is negatively coupled 
Table. Functional characterisation of regulatory molecules acting on pancreatic acinar cells

$1 \mathrm{Ca}^{2+}$ - Diacylglcerol mediated

CCK, gastrin, caerulein

muscarinic cholinergic

GRP, neuromedin C, bombesin

Substance $\mathrm{P}$, physalaemin

2 Cyclic AMP mediated

secretin

VIP

PHI

CGRP

somatostatin
3 Tyrosine Kinase Activating

insulin

IGF

EGF

4 Steroids

glucocorticoids

oestrogens to adenylate cyclase through a unique guanine nucleotide binding protein $\left(G_{i}\right)$. Finally, it is worth reiterating that while these agents have been mostly studied as regards secretion some are also known to increase cellular metabolism or protein synthesis and CCK, secretin and possibly cholinergic agents and bombesin affect cell growth in vivo. ${ }^{13}$

The third class of regulators activate tyrosine kinases and most of their effects are on acinar cell metabolism and biosynthetic events, although they also may act to potentiate digestive enzyme secretion. ${ }^{4}$ In contrast with the first two groups of regulatory agents which, except for muscarinic cholinergic receptors, are primarily localised in gut and brain, the tyrosine kinase activating receptors are widely distributed on a number of cell types in the body. These receptors have been extremely well studied in other cells to the point that their gene and protein sequences have been elucidated. ${ }^{56}$ In all cases the receptor contains an extracellular glycosylated domain, a hydrophobic transmembrane domain and an intracellular segment containing both tyrosine kinase activity and a specific tyrosine phosphorylation site. In general the properties of these receptors in the pancreas appear similar to those on other, better studied, cell types.

The fourth class of regulators for which the least is known as regards pancreas specific functions, is the steriod hormones. While evidence exists that members of the first three classes interact initially with receptors on the basolateral plasma membrane, steroid hormones generally interact with specific intracellular receptors. Pancreatic cytosolic receptors have been described for glucocorticoids, oestrogens and androgens. ${ }^{78}$ Most are similar to those in other tissues although an atypical low affinity oestrogen binding site requiring a peptide cofactor has been described. ${ }^{9}$ The physiological relevance of steriod receptors to tissue specific pancreatic functions is less clear although glucocorticoids probably regulate amylase gene expression along with insulin.

A number of additional regulatory molecules may affect pancreatic acinar cell function in vivo. Some act to stimulate or potentiate secretion (neurotensin, dopamine, adrenaline) while others are inhibitory (pancreatic polypeptide, PYY, NPY). These agents, however, have not been generally shown to act directly on acinar cells nor have specific acinar cell receptors been detected. Therefore, their site as well as mechanism of action are unknown. Two possibilities for an indirect mechanism of action are by influencing either the release of pancreatic islet hormones or altering neurotransmitter release from intrapancreatic nerve endings. While there is at present little evidence linking these agents with pancreatic adaptation, the possibility should not be discounted.

\section{Ligand binding characteristics of membrane receptors}

While the presence of a specific receptor can be inferred from studies of the actions of agonists and antagonists, more direct information has been obtained from ligand binding studies. Except for the muscarinic cholinergic receptor where labelled antagonists such as quinuclidinyl benzilate (QNB) or Nmethylscopolamine have been used, all pancreatic acinar receptors have been characterised with radiolabelled agonists. In most cases the peptides have been labelled with radioiodine by oxidative labelling of tyrosine residues. For some peptides, which lack an appropriate tyrosine, a synthetic analog is used which behaves similarly to the native peptide - for example, $\mathrm{Tyr}^{4}$-bombesin.

Characterisation of pancreatic receptors by ligand binding has been mainly carried out using isolated acinar cells or acini or by use of crude or purified cellular membrane fractions. In most cases studies using intact cells are easier to relate to control of cell function as receptor occupancy and stimulation of function can be carried out under similar conditions. On the other hand studies with isolated membranes are more suited to biochemical characterisation of the receptor as complicating events such as ligand internalisation do not occur and the receptors can more easily be exposed to unphysiological conditions. The major types of information obtained from ligand 
binding studies include affinity, number of receptors and specificity for agonists and antagonists. Most of this information is obtained from competition inhibition paradigms where binding of a small fixed amount of labelled ligand is carried out in the presence of increasing quantities of unlabelled ligand, analogues or inhibitors. The data can then be fitted graphically or by computer to derive binding constants.

By the additional technique of autoradiography the sites of ligand binding - that is, functional receptors, can be determined. This can be carried out at the light microscopic level either on embedded and sectioned material or by binding directly to slide mounted frozen and dried tissue sections. These techniques yield the cellular site of binding and further subcellular localization can be obtained by electron microscopic autoradiography. This has allowed demonstration that the initial site of peptide receptor binding in acinar cells is the basolateral membrane domain and also that at physiological temperatures the bound hormone is subsequently internalised and localised within specific intracellular compartments. ${ }^{10}$ While internalisation is clearly involved in some cases with degradation of the regulatory molecule the possibility exists that internalised peptides could have direct actions. Less precise but easier demonstration of peptide hormone internalisation has been carried out using acid washing or trypsinisation to selectively remove exposed, surface bound ligand. These studies showing internalisation in acinar cells have been carried out with insulin, IGF II, EGF, CCK, and somatostatin $^{1112}$ and it seems likely that all peptide regulators are internalised although to varying extents.

These results showing ligand internalisation complicate the interpretation of binding data obtained using intact cells at physiological temperature. Thus most of the data deriving number of functional receptors per cell probably is inaccurate. Other factors such as receptor internalisation also complicate this issue. In the case of CCK a single class of binding sites is observed on isolated plasma membranes while an additional class of higher affinity sites is observed on intact cells ${ }^{13}$ which is dependent on physiologic temperatures and cellular metabolic energy (J A Williams, unpublished data). With other ligands, such as insulin, similar affinities are observed for intact cells and plasma membranes.

\section{Molecular characterisation of membrane receptors}

While ligand binding data provides functional information about the receptor, it alone can provide little direct information about the physical composition of the receptor. Such information is provided by covalent receptor labelling, purification and determination of protein and gene sequences, and by the use of specific antireceptor antibodies. Information of this type has recently been obtained for several tyrosine kinase receptors and for some neurotransmitter receptors. Little is yet known about the more tissue specific gut peptides which act as regulators of pancreatic function.

By use of bifunctional crosslinking agents or the labelling of peptide regulators with a photoactive group, proteins at or near the receptor binding site of several tissue specific pancreatic receptors have been characterised including those for CCK, VIP and somatostatin. ${ }^{101415}$ Evaluation on polyacrylamide gels reveals the size and disulfide bond linked subunit composition while solubilisation in nonionic detergents followed by endoglycosidase treatment or lectin chromatography provides information as to the carbohydrate content of the receptor. Such studies have shown the aforementioned peptide receptors to be glycoproteins with binding subunits ranging in size from 55 to 80 kilodaltons. Tyrosine kinase receptors on pancreas have been shown to be similar to better characterised receptors on other tissues and generally are larger and more complex than other types of peptide hormone receptors.

More complete characterisation can be provided by solubilisation and purification of functional receptor. This requires solubilisation of the receptor with retention of binding such that ligand binding can then be used to follow purification. For tissue specific pancreatic receptors this has been done for CCK only. ${ }^{16}$ Techniques used for other receptors such as lectin chromatography and ligand affinity chromatography should then be feasible and have been carried out for the CCK receptor (Williams et al, unpublished data).

The molecular approaches which have been used for EGF and insulin receptors can lead to a detailed knowledge of receptor structure, and in conjunction with the development and use of antireceptor antibodies, to knowledge of receptor biosynthesis and cellular localisation. This will ultimately be needed to understand the role of various receptors in growth and adaptation. Even in more amenable and better studied cells such as fibroblasts, however, it is not yet clear how these receptors induce growth. Further information should come from site directed nucleotide mutagenesis with production of altered cellular receptors.

\section{Regulation of membrane receptors}

The number of cellular receptors for a particular regulator is not fixed but can increase or decrease because of altered rates of receptor synthesis or 
degradation. The most common form of such regulation is 'down regulation' of a receptor by its ligand. In this phenomenon, receptor occupancy leads to internalisation and degradation with a subsequent reduced ability of the cell to respond to the regulator as a result of a decrease in functional receptor number. Thus the response to prolonged stimulation is limited. Such down regulation of acinar receptors has been shown in vitro for insulin and acetylcholine. ${ }^{1718}$

It has been observed recently that occupancy of a specific receptor can also affect the binding properties of another distinct class of receptors. On acinar cells, CCK and other $\mathrm{Ca}^{2+}$-DAG mediated agents decrease the binding of EGF, IGF-II and somatostatin. ${ }^{111219}$ Some of this effect is probably mediated by endogenous activation of protein kinase $\mathrm{C}$ and may well involve receptor phosphorylation. ${ }^{1920}$ In contrast, insulin increases the binding of IGF II to its receptor. ${ }^{12}$ The functional consequences of this heterologous receptor regulation are not clear at present.

Two cases are known in which acinar cell CCK receptors have been studied during periods of adaptation. In the period immediately after birth the rat pancreas responds poorly to CCK and only a few CCK receptors are present. ${ }^{21}$ Over the next few weeks CCK binding increases and concurrently the pancreas develops its ability to respond to CCK. In the other case, CCK receptor binding was studied in rat acini after the animals were treated acutely or chronically with CCK in a manner known to induce pancreatic growth. No receptor down regulation was observed and in fact when acini were prepared $2 \mathrm{~h}$ after injection, CCK binding was increased. ${ }^{22}$ After one week of chronic CCK injections, with attendant pancreatic hypertrophy and hyperplasia, the number of receptors per cell was normal although the total number of receptors per pancreas was increased. ${ }^{23}$ These changes may be important in the adaptive growth response as they allow sustained growth which might be shut off if down regulation occurred.

Further insight into receptor regulation on acinar cells clearly requires the application or development of techniques to look directly at rates of receptor synthesis, internalisation and degradation as opposed to only following ligand binding.

\section{Relation of receptors to the trophic response}

While it appears obvious that pancreatic receptors are involved in mediating the trophic or adaptive response initiated by extracellular regulators little is known concerning the detailed mechanisms. The first major question is how do receptors initiate trophic responses? This clearly involves an intracellular signal which could be one of the same intracellular mes- sengers that activates secretion such as $\mathrm{Ca}^{2+}, \mathrm{DAG}$ or cAMP. This would imply, however, that all regulatory agents acting through a common intracellular mechanism such as $\mathrm{Ca}^{2+}$-DAG should regulate growth in a similar manner. Recent studies in vitro using cultured pancreatic acinar cells suggests this may not be true.$^{24}$ If we are forced to consider a receptor specific mechanism this could indicate direct involvement of either internalised ligand or receptor. Alternatively some new as yet not understood mechanism of action might exist perhaps mediated by a member of the rapidly expanding class of guanine nucleotide binding or $G$ proteins.

The second major question is how do receptors change during a trophic response? If growth is initiated by a sustained increase in an extracellular regulatory signal, how do the receptors for that and other regulators adapt. As 'down-regulation' tends to damp out a response, do receptors for trophic stimuli not show this response? This may depend on whether the sustained growth is driven by a sustained extracellular regulatory signal or not. As the biological response is dependent on the receptor number as well as the concentration of extracellular regulator both homologous and heterologous regulation of receptors may be important in the trophic response.

Further work on the structure, mechanism of action and regulation of receptors is clearly necessary to understand adaptive as well as the acute functional response of the pancreas.

\section{References}

1 Solomon TE. Regulation of exocrine pancreatic cell proliferation and enzyme synthesis. In: Johnson LR ed. Physiology of the digestive tract. New York: Raven Press, 1981: 873-92.

2 Williams JA Hootman SR. Stimulus-secretion coupling in pancreatic acinar cells. In: Go VLW., Brooks F, DiMagno E, Gardner JD., Lebenthal E, Scheele G, eds. The exocrine pancreas: basic and clinical aspects. New York: Raven Press, 1986: 123-39.

3 Solomon TE, Vanier M, Morisset J. Cell site and time course of DNA synthesis in pancreas after caerulein and secretin. Am J Physiol 1983; 245: G99-G105.

4 Williams JA, Goldfine ID. The insulin-pancreatic acinar axis. Diabetes 1985; 34: 980-6.

5 Ullrich A, Coussens L, Mayflick JS et al. Human epidermal growth factor receptor cDNA sequence and aberrant expression of the amplified gene in A431 epidermoid carcinoma cells. Nature 1984: 309: 418-25.

6 Ullrich A, Bell JR, Chen EY et al. Human insulin receptor and its relationship to the tyrosine kinase family of oncogenes. Nature 1985; 313: 756-61.

7 Svec F, Rudis M. Glucocorticoid hormone receptors in rat pancreas. Biochim Biophys Acta 1981 ; 674: 30-6.

8 Sandbert AA, Rosenthal HE. Steroid receptors in 
exocrine glands: the pancreas and prostate. J Steroid Biochem 1979; 11 : 293-9.

9 Boctor AM, Band P, Grossman A. Requirement for an accessory factor for binding of $\left[{ }^{3} \mathrm{H}\right]$ estradiol to protein in the cytosol fraction of rat pancreas. Proc Natl Acad Sci USA 1981; 78: 5648-51.

10 Goldfine ID, Williams JA. Receptors for insulin and CCK in the acinar pancreas: relationship to hormone action. Int Rev Cytol 1983; 85: 1-38.

11 Logsdon CD, Williams, JA. Intracellular $\mathrm{Ca}^{2+}$ and phorbol esters synergistically inhibit EGF internalization in pancreatic acini. Biochem J 1984; 223: 893-900.

12 Mossner J, Logsdon CD, Potau N, Williams JA, Goldfine ID. Effect of intracellular $\mathrm{Ca}^{2+}$ on insulin-like growth factor II internalization into pancreatic acini: role of insulin and cholecystokinin. J Biol Chem 1984; 259: $12350-6$.

13 Sankaran H, Goldfine ID, Deveney CW, Wong KY, Williams JA. Binding of cholecystokinin to high affinity receptors on isolated rat pancreatic acini. J Biol Chem 1980; 255: 1849-53.

14 Sakamoto C, Goldfine ID, Williams JA. The somatostatin receptor on isolated pancreatic acinar cell plasma membranes: identification of subunit structure and direct regulation by cholecystokinin. J Biol Chem 1984; 259: 9623-7.

15 Rosenzweig SA, Miller LJ, Jamieson JD. Identification and localization of cholecystokinin-binding sites on rat pancreatic plasma membranes and acinar cells: a biochemical and autoradiographic study. J Cell Biol 1983; 96: 1288-97.

16 Szecowka J, Goldfine ID, Williams JA. Solubilization and characterization of active CCK receptors from mouse pancreas. Regulatory Peptides 1985; 10: 71-83.

17 Mossner J, Logsdon CD, Goldfine ID, Williams JA. Regulation of pancreatic acinar cell insulin receptors by insulin. Am J Physiol 1984; 247: G155-60.

18 Hootman SR, Brown ME, Williams JA, Logsdon CD. Regulation of muscarinic acetylcholine receptors in cultured guinea pig pancreatic acini Am J Physiol 1986; 251 : G75-83.

19 Matozaki T, Sakamoto C, Nagao M, Baba S. Phorbol ester or diacylglycerol modulates somatostatin binding to its receptors on rat pancreatic acinar cell membranes. J Biol Chem 1986; 261 : 1414-20.

20 Davis RJ, Czech MP. Tumor-promoting phorbol diesters cause the phosphorylation of epidermal growth factor receptors in normal human fibroblasts at threonine-654. Proc Natl Acad Sci USA 1985; 83: 1974-8.

21 Leung YK, Lee PC, Lebenthal E. Maturation of cholecystokinin receptors in pancreatic acini of rats. $\mathrm{Am}$ J Physiol 1986; 250: G594-G597.

22 Otsuki M, Okabayashi Y, Ohki A, Hootman SR, Baba $\mathrm{S}$, Williams JA. Amylase secretion by isolated pancreatic acini after acute cholecystokinin treatment in vivo. $\mathrm{Am} \mathrm{J}$ Physiol 1984; 246: G419-25.

23 Otsuki M, Williams JA. Altered amylase secretion by isolated pancreatic acini following in vivo administration of cholecystokinin. Am J Physiol 1983; 244: G683-8.

24 Logsdon CD. Regulation of pancreatic acinar cell growth: effects of cholecystokinin, EGF and insulin in vitro. Am J Physiol 1986; 251: G487-940. 